\title{
Advances in Robotic FUE
}

\author{
Robert M. Bernstein, MD I New York, New York, USA I rbernstein@bernsteinmedical.com; \\ Michael B. Wolfeld, MD I New York, New York, USA
}

\begin{abstract}
Disclosure: Drs. Bernstein and Wolfeld hold equity interest in Restoration Robotics, Inc. Dr. Bernstein is on its medical advisory board.
\end{abstract}

Since the publication of "What's New in Robotic Hair Transplantation" (Hair Transplant Forum Int'l. 2017; 27(3):100101), there have been important improvements to the robotic system in both its incision and recipient site creation capabilities. These advances fall into four overlapping categories: increased speed, increased accuracy, increased functionality, and improved artificial intelligence (AI). The overlap occurs since improvements in functionality, accuracy, and Al can also increase the overall speed of the procedure. A faster procedure decreases the time grafts are outside the body and allows the physician to perform larger cases without placing additional oxidative stress on the follicles.

\section{Increased Speed}

The speed of the robot has increased through faster and more precise alignment with the hair in the follicular units. The robot also saves a significant amount of time by staying closer to the scalp (approximately $2 \mathrm{~mm}$ ) while moving from unit to unit, rather than retracting after each harvest. By shortening the distance the robotic arm moves between incisions, the dissection cycle has decreased to less than 2 seconds, giving the robot a raw speed over 2,000 grafts per hour. In a clinical setting, this enables harvesting of up to 1,300 grafts per hour.

Although the obvious way to increase speed is to simply make the robot go faster, there are limitations to this, as it would decrease the ability of physicians to make real-time adjustments to the system. The robot has an automatic feedback loop that makes intra-operative modifications as the harvesting proceeds, and this significantly decreases the need for human intervention. However, when there is scarring or other situations of excessive patient variability, it is necessary for occasional "tweaking" (particularly of punch depth) to achieve an optimal outcome. In these situations, faster robot speed may be counterproductive.

With this in mind, new ways have been found to speed up the procedure without limiting the operator's ability to respond. One has been to change the color of the light emitted by the optical system. In the past, a beam of red light illuminated the fiducials that the robot uses to guide the robotic arm, but the glare of this light is very difficult on the eyes. By enabling the optical system to read "eye-friendly" white light, the surgical team is now able to remove grafts as soon as they are separated from the surrounding tissue, rather than having to wait for an entire grid to be finished. This allows the two steps in follicular unit excision-the graft separation from surrounding tissue (incision) and the actual removal (extraction) - to proceed in parallel, rather than in series, in order to decrease operating time.

The new optical system also enables the robot to recognize the tensioner from a distance. Previously, the physician had to manually bring the robot toward the scalp (a step called "forced drag"), until the robot was close enough to recognize the fiducials on a grey-colored tensioner. This now happens automatically, with the robot recognizing a yellow tensioner from a distance and then homing in on the fiducials as it moves closer to the scalp, eliminating the time needed for the extra step (Figure 1).

Recipient site creation has been a significant new capability of the robotic system. The advantages of robotic site creation include the ability to avoid existing terminal hair (minimizing injury) and to create new recipient sites in a precise distribution that complements the existing hair. A limitation of this technology is that the physician needs to develop a 3-D computer-based model of each patient's scalp to communicate the transplant design to the robot. The old model required the fusion of 5 two-dimensional images, a process that required a significant amount of time. The newest iteration can build a three-dimensional model using only one image, greatly decreasing the time needed for this important step (Figure 2).

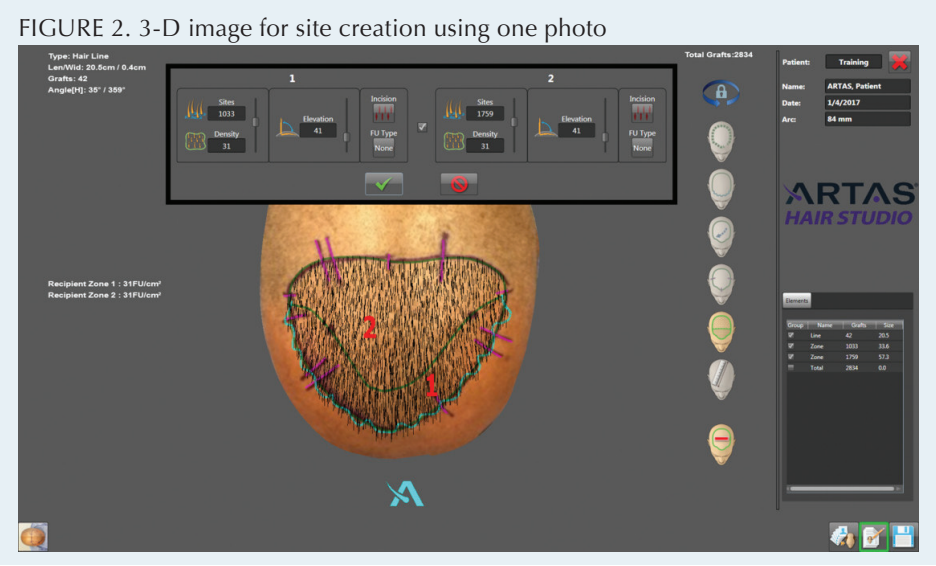

\section{Increased Accuracy}

There has been a recent trend in FUE towards using smaller punches. Although these authors feel that in many cases the increased risk of transection from smaller diameter punches outweighs the benefit of reduced wounding and concomitant smaller scars, it is important that the robot has this capability for physicians who prefer these punches. 
FIGURE 3. 1.0, 0.9 and $0.8 \mathrm{~mm}$ needles

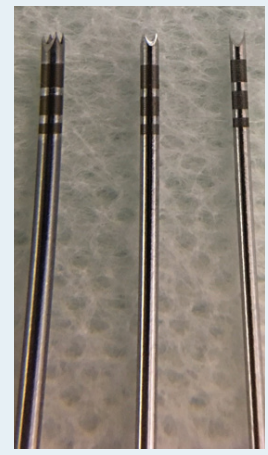

The sharp/blunt system in the original robot (released in 2011) used a $1.0 \mathrm{~mm}$ sharp pronged needle that penetrated the skin about $1 \mathrm{~mm}$ and was immediately followed by a rotating, dull punch with a slightly larger diameter that went deeper into the scalp. The current system includes a $0.9 \mathrm{~mm}$ needle that is the workhorse for most cases. With refinements in the optical system, the needle/punch diameter was able to be reduced further. The new needle option is $0.8 \mathrm{~mm}$.

The needle has also been redesigned so that the physician can choose between 2 and 4 prongs, with the former being preferable in softer tissue and the latter in firmer skin or scarred scalp (Figures 3 through 6).

FIGURE 4. Recipient wounds: $0.8 \mathrm{~mm}$ (left) and $0.9 \mathrm{~mm}$ (right)

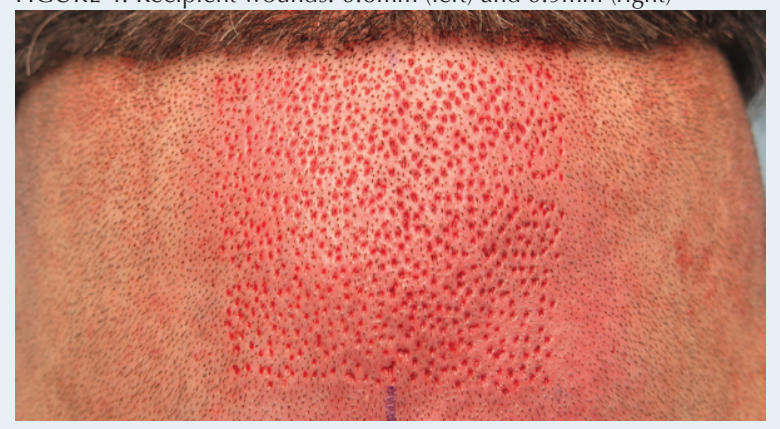

FIGURE 5. 0.8mm needle: 1-, 2-, 3-, and 4-hair follicular unit grafts

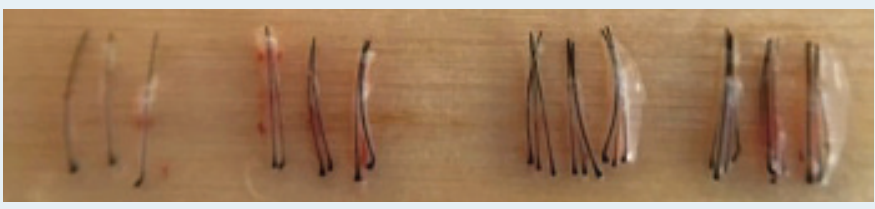

FIGURE 6. 0.9mm needle: 1-, 2-, 3-, and 4-hair follicular unit grafts

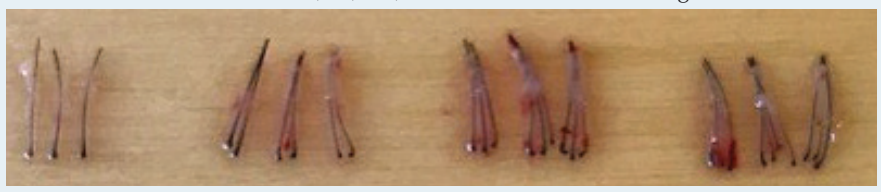

\section{Increased Functionality}

In prior iterations, when the robotic arm was in a position that was too cramped and from which it could not automatically recover, the user needed to go through a six-step manual process using a stand-alone pendant to guide the robot to a neutral "safe" position. The Arm Brake Release is a new functionality that places a single button on the arm that, when pressed, quickly moves the arm away, allowing the operator to readjust the patient's position.

Modifications of the robotic arm (which give it greater reach) and changes to the robotic head (which reduce its bulk) enable the robot to access a much greater area of the scalp without the need for repositioning the patient. This reduces a significant amount of procedural time as well. Another advantage of the smaller head is that the robotic arm can approach the patient at more acute angles without collision, adding more flexibility to both harvesting and site creation (harvesting to $35^{\circ}$, site making to $30^{\circ}$ ). The more acute angles required a redesign of the headrest so that the arm would have unimpeded access to the scalp (Figure 7 ).

Prior iterations of the robotic system used hypodermic needles of varying sizes (18g-21g) for recipient site making. In response to the wide range of physician preferences, the robot now has a universal holder that can accommodate almost any type of site making tool. These include square-tipped blades, angled blades, and chisel and spear point blades, as well as the original hypodermic needles. These can be easily interchanged during the procedure (Figure 8).

\section{Artificial intelligence}

An automatic collision recovery system will automatically retract the robotic arm if the arm approaches the patient at an angle that is too acute, or cramped to operate, or if any part of the robot (other than the operating tip) inadvertently touches the patient. Once retracted, the patient can be repositioned so that the FUE session can proceed.

One of the frustrations of FUE is the occasional empty site that represents either a graft that was pushed too deeply into the scalp or one that was completely removed. The new empty site warning icons complement physician observation by using color-coded symbols (green, yellow, and red) to alert the doctor to the occurrence of empty sites.

Finally, the ARTAS software can now automatically detect regions with low (or no) hair density and block those areas from being harvested. This capability decreases human error and saves time by automatically performing a function that prevents creating zones with very little or no hair coverage (Figure 9).

In sum, new improvements in the speed, functionality, accuracy, and

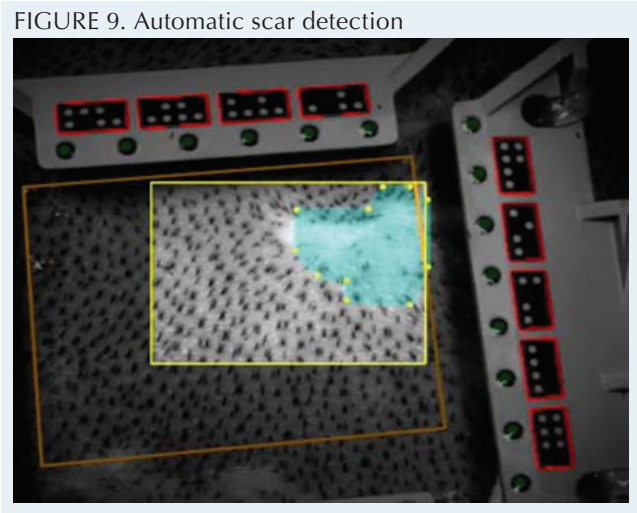
artificial intelli-

gence of the robotic system have significantly shortened the duration of the overall procedure. Besides being more convenient for patients and more expedient for the operating physician, the shortened operating time decreases the time grafts are outside the body, an important factor in ensuring optimal growth of the transplanted hair. 